\title{
Towards a dynamical mass of a PG 1159 star: radial velocities and spectral analysis of SDSS J212531-010745
}

\author{
B. Beeck, ${ }^{1}$ S. Schuh, ${ }^{1}$ T. Nagel, ${ }^{2}$ and I. Traulsen ${ }^{1}$ \\ ${ }^{1}$ Institut für Astrophysik, Universität Göttingen, Friedrich-Hund-Platz 1, 37077 Göttingen, Germany \\ 2 Institut für Astronomie und Astrophysik, Universität Tübingen, Sand 1, 72076 Tübingen, Germany
}

\begin{abstract}
The evolutionary scenarios which are commonly accepted for PG 1159 stars are mainly based on numerical simulations, which have to be tested and calibrated with real objects with known stellar parameters. One of the most crucial but also quite uncertain parameters is the stellar mass. PG 1159 stars have masses between 0.5 and $0.8 \mathrm{M}_{\odot}$, as derived from asteroseismic and spectroscopic determinations. Such mass determinations are, however, themselves model-dependent. Moreover, asteroseismically and spectroscopically determined masses deviate systematically for PG 1159 stars by up to $10 \%$.

SDSS J212531.92-010745.9 is the first known PG 1159 star in a close binary with a late-main-sequence companion allowing a dynamical mass determination. We have obtained 14 Calar Alto spectra of SDSS J212531.92-010745.9 covering the full orbital phase range. A radial velocity curve was extracted for both components. With co-added phasecorrected spectra the spectral analysis of the PG 1159 component was refined. The irradiation of the companion by the PG 1159 star is modelled with PHOENIX, yielding constraints on radii, effective temperature and separation of the system's components. The light curve of SDSS J212531.92-010745.9, obtained during three seasons of photometry with the Göttingen $50 \mathrm{~cm}$ and Tübingen $80 \mathrm{~cm}$ telescopes, was modelled with both the nightfall and PHOEBE programs.
\end{abstract}

Individual Objects: SDSS J212531.92-010745.9

\section{Extraction of the radial velocity curves}

In August 2007, 14 spectra of SDSS J212531.92-010745.9 covering the total phase range were taken with the TWIN spectrograph at the $3.5 \mathrm{~m}$ telescope at Calar Alto Observatory (Alméria, Spain). These show typical PG 1159 features together with the Balmer series of hydrogen in emission (plus other emission lines), already interpreted as signature of an irradiated close companion by Nagel et al. (2006) from an SDSS spectrum. The Calar Alto spectra cover wavelengths from $3800 \AA \AA$ up to about $7000 \AA ̊$. At $5000 \AA$ they have a resolution of $R=4170$ and a SNR ranging from 4 to 13 . The spectra were reduced and normalized to their continua. To determine the radial velocity (RV) curve of the secondary of the system a Gaussian was fitted to seven $\mathrm{H}$ Balmer lines $(\mathrm{H} \alpha, \mathrm{H} \beta, \ldots, \mathrm{H} \eta$, emission line height variable with phase) to locate the line centres. To evade uncertainties in the wavelength calibration and intrinsic wavelength dependence of the RV, a sine was fitted to the RV curve separately for each line. The zero points of the fits were shifted to the mean zero point $\left\langle v_{0}\right\rangle=81.5 \mathrm{~km} \mathrm{~s}^{-1}$ with an uncertainty of $\pm 9.4 \mathrm{~km} \mathrm{~s}^{-1}$. The weighted 


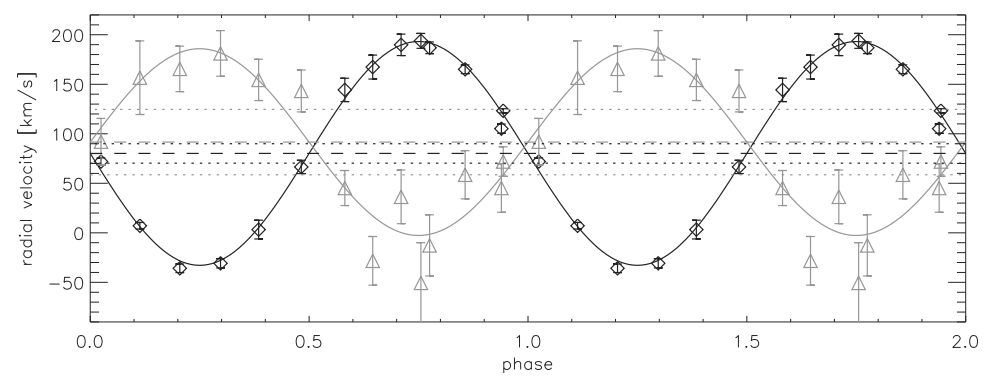

Figure 1: Radial velocities (RVs) derived from the Calar Alto spectra. The grey symbols and error bars signify the RVs of the PG 1159 component together with the sine fit (solid grey line; amplitude $\left.K_{1}=(94.3 \pm 15.0) \mathrm{km} \mathrm{s}^{-1}\right)$, the zero point of which is shown as dashed line. The black symbols signify the RVs of the secondary, again with the sine fit (solid black line; amplitude $K_{2}=(113.0 \pm 3.0) \mathrm{km} \mathrm{s}^{-1}$ ) and its zero point (dashed black line). The dotted lines are the uncertainties of the zero points.

mean of the shifted RVs was calculated and a sine was fitted to the resulting mean RVs yielding a secondary RV amplitude of $K_{2}=(113.0 \pm 3.0) \mathrm{km} \mathrm{s}^{-1}$. The primary RV curve was deduced from the cross correlation of a small section of the spectra containing two narrow CIV absorption lines at $5801 / 5812 \AA$, which were unblended with the strong emission lines of the companion. The accuracy of the RV values obtained by this method was estimated to amount to $\pm 30 \mathrm{~km} \mathrm{~s}^{-1}$ and was rescaled with the SNR of the individual spectra. Again a sine was fitted to the RVs. The amplitude of this fit is $K_{1}=(94.3 \pm 15.0) \mathrm{km} \mathrm{s}^{-1}$ and its zero point is at $v_{0, P G 1159}=(92 \pm 33) \mathrm{km} \mathrm{s}^{-1}$, slightly redshifted with respect to the zero point of the RV curve of the secondary. Although this redshift is insignificant, it raises the hope to detect a gravitational redshift for the PG 1159 component of SDSS J212531.92-010745.9 with high-resolution spectroscopy in subsequent studies. The RV curves are shown in Fig. 1.

\section{Spectral analysis and light curve modelling}

Using the RV curves obtained, the spectra were shifted to zero redshift for the individual components of the system to determine a mean and median of both the primary and secondary spectrum. A grid of model spectra was computed with the non-LTE atmosphere modelling package NGRT (Werner et al. 2003) and fitted to the median primary spectrum. The best fit so far is obtained for a model with $T_{\text {eff }}=72500 \mathrm{~K}, \log g=7.20$ and abundances (by number) of $\mathrm{C} / \mathrm{He}=0.07, \mathrm{~N} / \mathrm{He}=0.01, \mathrm{O} / \mathrm{He}=0.01$, implying a mass fraction $(\mathrm{He} / \mathrm{C} / \mathrm{N} / \mathrm{O})=(0.78 / 0.16 / 0.03 / 0.03)$, typical for PG 1159 stars - see e. g. Werner \& Herwig (2006). Typical errors for this kind of analysis are $\pm 10000 \mathrm{~K}$ for $T_{\text {eff }}$ and \pm 0.3 for $\log g$ (Ziegler et al. 2009). The temperature obtained is significantly lower than the one found by Nagel et al. (2006), who obtained the preliminary values $T_{\text {eff }}=90000 \mathrm{~K}$ and $\log g=7.60$, and implies that SDSS J212531.92-010745.9 is one of the coolest PG 1159 stars.

To get the stellar parameters of the irradiated secondary, a second model grid is being calculated using PHOENIX (Hauschildt et al. 1997). Up to now, no quantitative result has been obtained, but the Balmer series of hydrogen in emission is reproduced by the first models calculated.

Three seasons of photometry are available for SDSS J212531.92-010745.9. The system shows flux variations with a peak-to-peak amplitude of about $0.7 \mathrm{mag}$ and a period of about $6.96 \mathrm{~h}$. The ephemeris could be determined to a high accuracy. The light curve profile 
obtained shows no eclipse and is currently being fitted with the binary modelling programs nightfall and PHOEBE (Prša \& Zwitter 2005). This light curve modelling possibly constrains the inclination of the system which is needed to deduce the mass of the components from the RVs. Together with the constraints of an optimized spectral analysis (especially with PHOENIX) this will give a possible mass range for the PG 1159 component of SDSS J212531.92-010745.9 (work in progress: Beeck 2009, Schuh et al. 2009, and references therein).

Acknowledgments. The spectroscopy is based on service observations collected by J. Aceituno and U. Thiele at the Centro Astronómico Hispano Alemán, operated jointly by the MaxPlanck Institut für Astronomie and the Instituto de Astrofísica de Andalucía. Special thanks to B. Gänsicke and M. Schreiber who first directed our attention to this unique object, and to all observers for the photometric observations. We also thank the Astronomische Gesellschaft as well as the conference sponsors and in particular HELAS for financially supporting the poster presentation at JENAM 2008 through travel grants to B.B. and S.S.

\section{References}

Beeck, B. 2009, Diploma thesis, University of Göttingen, in prep.

Hauschildt, P., Baron, E., \& Allard, F. 1997, ApJ, 490, 803

Nagel, T., Schuh, S., Kusterer, D.-J., et al. 2006, A\&A, 448, L25

Prša, A., \& Zwitter, T. 2005, ApJ, 628, 426

Schuh, S., Beeck, B., \& Nagel, T. 2009, in "White Dwarfs", J. Phys.: Conf. Ser., in press, arXiv:0812.4860

Werner, K., Deetjen, J. L., Dreizler, S., et al. 2003, in "White Dwarfs", NATO ASIB Proc., 105, 117

Werner, K., \& Herwig, F. 2006, PASP, 118, 183

Ziegler M., Rauch, T., Werner, K., Koesterke, L., Kruk, J. W., 2009, CoAst, 159, 107

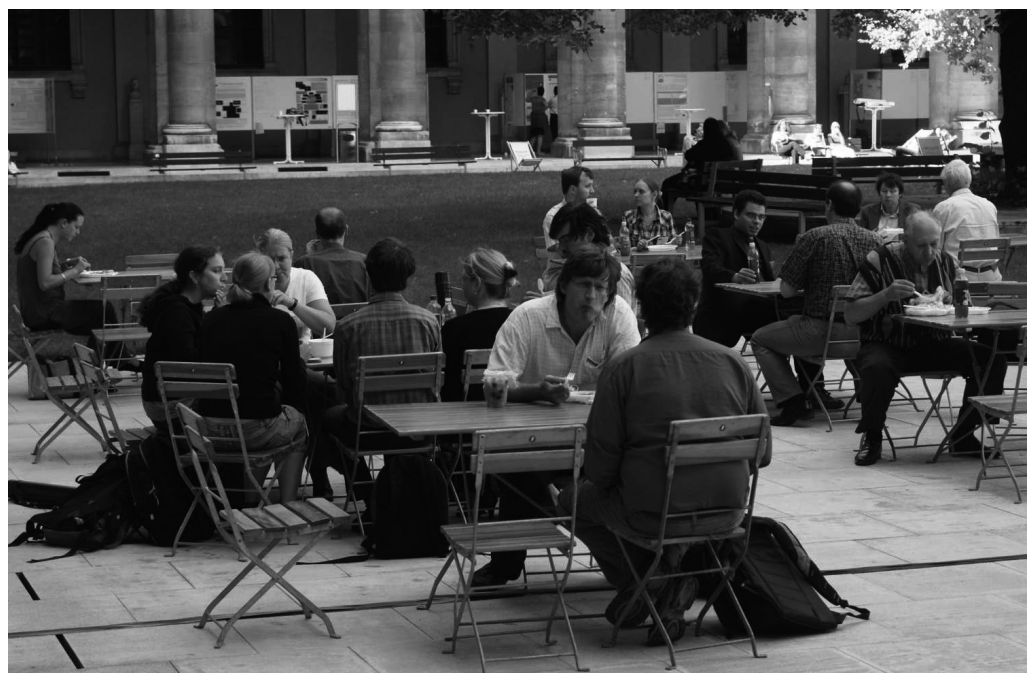

